Research Paper

\title{
Nab-paclitaxel in combination with Bevacizumab in patients with non-squamous non-small cell lung cancer after failure of at least one prior systemic regimen
}

\author{
Xuezhi Hao ${ }^{1}$, Yixiang Zhu ${ }^{1,2}$, Yuxin $\mathrm{Mu}^{1}$, Shouzheng Wang ${ }^{1}$, Junling $\mathrm{Li}^{1}$, Puyuan Xing ${ }^{1 凶}$ \\ 1. National Cancer Center/National Clinical Research Center For Cancer/Cancer Hospital, Chinese Academy of Medical Science and Peking Union Medical \\ College, Beijing, 100021, China. \\ 2. Affiliated Hospital of Guizhou Medical University, Guizhou Province Tumor Hospital, Guiyang, P.R. China.
}

$\triangle$ Corresponding author: Puyuan Xing, National Cancer Center/National Clinical Research Center for Cancer/Cancer Hospital, Chinese Academy of Medical Science and Peking Union Medical College, Beijing, 100021, China. Tel: +86 13521005935; Fax: +86-21-57643271; E-mail: xingpuyuan@cicams.ac.cn.

(c) The author(s). This is an open access article distributed under the terms of the Creative Commons Attribution License (https://creativecommons.org/licenses/by/4.0/). See http:/ /ivyspring.com/terms for full terms and conditions.

Received: 2020.04.15; Accepted: 2020.07.10; Published: 2020.09.13

\begin{abstract}
Background: Most patients with non-small cell lung cancer (NSCLC) experience disease progression after first-line treatment. The efficacy and safety of the nab-paclitaxel (nab-PTX) and bevacizumab combination as the second or further line of treatment in patients with advanced NSCLC have not been reported yet.

Objective: To evaluate the efficacy and safety of the nab-PTX and bevacizumab combination in patients with advanced non-squamous (NSQ) NSCLC after failure of at least one prior systemic regimen.

Methods: Patients with advanced (stage IV) NSQ NSCLC who received the nab-PTX and bevacizumab combination as the second or further line treatment between February 2012 and December 2018 at the Cancer Hospital of the Chinese Academy of Medical Sciences (Beijing, China) were included in this retrospective study. The main outcomes included the objective response rate (ORR), progression-free survival (PFS), overall survival (OS), and safety.

Results: Thirty-four patients received 1-27 cycles (median, four cycles) of treatment; 67.6\% (23/34) patients had undergone at least two lines of previous treatment. The ORR and disease control rates were $26.5 \%(9 / 34)$ and $82.4 \%(28 / 34)$, respectively. The median PFS and OS were $6.0(95 \% \mathrm{Cl}=2.9-7.2)$ and 11.0 ( $95 \% \mathrm{Cl}=7.8-18.7)$ months, respectively. The multivariable analyses indicated that the combined use of other drugs and pleural metastasis were respectively associated with better PFS (hazard ratio $=0.354,95 \%$ $\mathrm{Cl}=0.134-0.935, P=0.036$ ) and $\mathrm{OS}$ (hazard ratio $=0.540,95 \% \mathrm{Cl}=0.118-0.980, P=0.046$ ). The most frequent grade 3-4 adverse events (AEs) were neutropenia $20.6 \%$ (7/34), leukopenia $8.8 \%(3 / 34)$, and anemia $5.9 \%(2 / 34)$. No grade 5 AE occurred.

Conclusion: Combined nab-PTX and bevacizumab might be an effective treatment regimen for patients with advanced NSQ NSCLC after failure of at least one prior systemic regimen, but studies have to validate those findings.
\end{abstract}

Key words: carcinoma, non-small cell lung; nab-paclitaxel; bevacizumab; survival; adverse events

\section{Introduction}

Non-small cell lung cancer (NSCLC) is the most common type of lung cancer [1,2]. NSCLC is often diagnosed at an advanced stage [3-5]. Tyrosine kinase inhibitors (TKIs) targeting the epidermal growth factor receptor (EGFR) or anaplastic lymphoma kinase (ALK) have improved the overall survival (OS) of patients with the corresponding genetic alterations [6-9]. Immune checkpoint inhibitors such as nivolumab or pembrolizumab as first-line treatments also significantly prolonged patient OS [10-12]. Nevertheless, despite the efficacy of those drugs, treatment will eventually fail, the disease will 
progress [13], and the patients will need second and further lines of treatment $[14,15]$. The prognosis of patients with advanced NSCLC remains poor, especially for patients who received multiple lines of treatment $[1,2,16]$.

Nab-paclitaxel (nab-PTX) is a nanoparticle formulation of paclitaxel bound to human serum albumin [17, 18]. Compared with solvent-based paclitaxel (sb-PTX), nab-PTX can enhance drug delivery of the cytotoxic agent to tumors, increase the intra-tumor drug concentration $[19,20]$, and minimize the occurrence of hypersensitivity reactions due to the absence of the culprit solvent [21-23]. The phase III open-label CA031 trial demonstrated that nab-PTX achieved a higher objective response rate (ORR) (33\% vs. $25 \%$ ) and a lower occurrence of grade 3-4 neutropenia ( $47 \%$ vs. $58 \%$ ) compared with sb-PTX as first-line treatment for NSCLC [24]. Based on the above results, the US FDA approved nab-PTX in combination with carboplatin for the first-line treatment of locally advanced or metastatic NSCLC. Real-world evidence also suggested that first-line treatment with nab-PTX plus carboplatin prolonged progression-free survival (PFS) and OS in patients with NSCLC $[25,26]$. In China, nab-PTX is mostly used as the second or further line treatment option because of limitations in indications, medical insurance, and costs, among others. A few studies reported that nab-PTX as a second or further line chemotherapy regimen was effective in advanced NSCLC in Western and East Asian populations [27-29]. Nevertheless, there is no consensus or standardized protocol for the second or further line use of nab-PTX in patients with advanced NSCLC, and the exact effectiveness and safety data of nab-PTX in unknown.

Bevacizumab is a monoclonal antibody that inhibits angiogenesis by targeting the vascular epidermal growth factor (VEGF) [30]. The addition of bevacizumab to carboplatin/paclitaxel (solvent-based chemotherapy) as a first-line regimen showed clinical benefits in the treatment of advanced NSCLC in several randomized controlled trials (RCTs) and has been recommended for patients without contraindications [31]. The IFCT-1103 ULTIMATE trial showed that weekly sb-PTX with bevacizumab was superior to docetaxel as second- and third-line therapy for advanced NSCLC [32].

There is still a lack of evidence for the use of nab-PTX in combination with bevacizumab for the second or further line of treatment in patients with advanced NSCLC. Therefore, the aim of this study was to evaluate the efficacy and safety of the nab-PTX in combination with bevacizumab in patients with advanced non-squamous (NSQ) NSCLC after failure to at least one prior systemic regimen.

\section{Materials and Methods}

\section{Study design and patients}

This was a retrospective study of the patients with advanced (stage IV) NSCLC who received nabPTX and bevacizumab as second or further line treatment between February 2012 and December 2018 at the Cancer Hospital of the Chinese Academy of Medical Sciences (CAMS) (Beijing, China). The study was approved by the Ethics Committee of the Cancer Hospital of the CAMS (Beijing, China) (approval number: 15-144/1071).

The inclusion criteria were: 1) received at least one line of systemic therapy for stage IV/metastatic NSCLC (either chemotherapy or TKIs); 2) received nab-PTX and bevacizumab in later-line regimen, for at least one cycle with radiological evaluation; 3 ) $\geq 18$ years of age; 4) Eastern Cooperative Oncology Group (ECOG) performance status (PS) of 0-2; 5) pathologically confirmed non-squamous NSCLC; and 6) at least one measurable lesion according to RECIST 1.1. Patients who received a prior taxane were eligible except for nab-paclitaxel. The exclusion criterion was any contraindication to nab-paclitaxel or bevacizumab.

\section{Treatment}

The 130-nm albumin-bound formulation of paclitaxel (nab-PTX [Abraxane]; Celgene, Summit, NJ, USA; $100 \mathrm{mg} / \mathrm{vial}$ ) was routinely given at $130 \mathrm{mg} / \mathrm{m}^{2}$ over $30 \mathrm{~min}$ on days 1 and 8 while bevacizumab was given at a dose of $5-10 \mathrm{mg} / \mathrm{kg}$ on day 1 of a 21 -day cycle. The patients were scheduled to receive at least two cycles, and the therapeutic efficacy was evaluated every two cycles. Patients with symptom aggravation after one cycle were also evaluated. Treatment was discontinued if progressive disease (PD) or intolerable adverse events (AEs) occurred.

\section{Data collection}

The baseline patient data included age, sex, Eastern Cooperative Oncology Group (ECOG) performance status (PS), smoking history, stage, pathological type, number of previous treatment lines, number of treatment cycles of nab-PTX, prior taxane treatment, EGFR/ALK-mutation status, prior EGFR/ALK TKI treatment, and lung radiotherapy. PS was defined according to the ECOG performance scale [33]. All tumor staging procedures were carried out using the 7th Union for International Cancer Control tumor node metastasis (TNM) classification. 


\section{Treatment response and AEs}

Tumor response was assessed by computed tomography (CT) scan, magnetic resonance imaging (MRI), bone scan, and tumor markers after every two treatment cycles. The Response Evaluation Criteria in Solid Tumors (RECIST) 1.1 criteria were used for treatment response evaluation in terms of complete response (CR), partial response (PR), stable disease (SD), PD, ORR (as CR+PR), and disease control rate (DCR) (as $\mathrm{CR}+\mathrm{PR}+\mathrm{SD}$ ). Any $\mathrm{AE}$ that occurred between the initiation of treatment and until 1 month after ending treatment was recorded as an $\mathrm{AE}$, regardless of whether the $\mathrm{AE}$ was associated with the drug. The evaluation of AEs was based on the National Cancer Institute-Common Toxicity Criteria (NCI-CTC) 3.0 version.

\section{Outcomes}

The main outcomes included the ORR, PFS, OS, and AEs. These data were obtained from multiple sources, including clinical letters, follow-up examinations, and hospital computer information systems. PFS was determined as the time from the start of treatment to disease progression (local, regional, and/or distant) or death from any cause, whichever occurred first. OS was defined as the time from the start of treatment to death from any cause.

\section{Statistical analysis}

The patients' characteristics and treatment responses were analyzed using descriptive statistics. Continuous variables are presented as means \pm standard deviation and were analyzed using the Student t-test. Categorical variables are presented as frequencies and were analyzed using the chi-square test. PFS and OS were calculated with the Kaplan-Meier method. The frequencies of AEs were summarized using absolute frequencies and percentages. The Cox proportional hazard regression model was used to identify the risk factors associated with disease progression. In univariable analyses, all baseline variables were examined for association. In the multivariable analyses, the factors with $P$-values $<0.20$ in the univariable analyses were included. All statistical analyses were performed using SPSS 17 (IBM, Armonk, NY, USA). $P$ values $<0.05$ were considered statistically significant.

\section{Results}

\section{Characteristics of the patients}

From February 2012 to December 2018, 34 patients received 1-27 cycles (median, four cycles) of treatment. Their characteristics are listed in Table 1. Among them, $28(82.4 \%)$ patients were $<65$ years of age; five (14.7\%) were ECOG PS 0 and 29 (85.3\%) were PS 1; $21(61.8 \%)$ were male; and $15(44.1 \%)$ had a history of smoking. Most patients $(n=32 ; 94.1 \%)$ had adenocarcinoma; $12(35.3 \%)$ patients had confirmed EGFR mutation, while two $(5.9 \%)$ had ALK translocation. The brain and pleural metastasis rates were $26.5 \%(\mathrm{n}=9)$ and $50.0 \% \quad(\mathrm{n}=17)$, respectively. Regarding previous treatments, $16(47.1 \%)$ patients were treated with a taxane and $16(47.1 \%)$ with an EGFR/ALK TKI; 23 (67.6\%) patients had received one or two lines of chemotherapy prior to nab-PTX/ bevacizumab, and $23(67.6 \%)$ patients had received radiotherapy. Twelve patients received other drugs in addition to nab-PTX and bevacizumab: one $(2.9 \%)$ received sunitinib, two $(5.9 \%)$ received gemcitabine, two $(5.9 \%)$ received immune checkpoint inhibitors, and seven $(20.6 \%)$ received platinum. The median follow-up for all patients was 6.8 months without censoring or was not reached when using the reverse Kaplan-Meier method.

\section{Tumor response and survival}

As shown in Table 2, the ORR and DCR were $26.5 \%(9 / 34)$ and $82.4 \%(28 / 34)$, respectively. CR was not observed, while the PR rate was $26.5 \%(9 / 34)$. The SD and PD rates were 55.9\% (19/34) and 17.6\% (6/34), respectively.

The Kaplan-Meier analysis (Figure 1) showed that the median PFS and OS were 6.0 (95\% CI=2.9-7.2) and 11.0 (95\% CI=7.8-18.7) months, respectively. The 1-year PFS rate and 1-year OS rates were 10\% and $40 \%$, respectively, while the 2-year OS rate was $30 \%$.

\section{Factors associated with PFS and OS}

As shown in Table 3, in the univariable analyses for PFS and OS, only pleural metastasis $(P=0.029)$ was significantly associated with OS. In the multivariable analyses, the combined use of other drugs was associated with better PFS (HR=0.354, $95 \% \mathrm{CI}=0.134-0.935, P=0.036$ ), and pleural metastasis was associated with better OS (HR=0.540, 95\% CI $=0.118-0.980, P=0.046$ ) (Table 4).

\section{AEs}

Regarding hematological AEs, the majority were of mild severity. The occurrence rates of grade 3-4 of anemia, neutropenia, leukopenia, and peripheral were $5.9 \%, 20.5 \%, 8.8 \%$, and $2.9 \%$, respectively. No other grade 3-4 hematologic or non-hematological AEs were observed. No grade $5 \mathrm{AE}$ occurred in the present study.

\section{Discussion}

The efficacy and safety of nab-PTX and bevacizumab as a second or further line of treatment 
in patients with advanced NSCLC have not been reported yet. Therefore, this study aimed to evaluate the efficacy and safety of nab-PTX in combination with bevacizumab in patients with advanced NSQ NSCLC after failure of at least one prior systemic regimen. The results strongly suggest that the combined nab-PTX and bevacizumab might be an effective treatment regimen for patients with advanced NSQ NSCLC after failure of at least one prior systemic regimen, but studies have to validate those findings. In addition, the toxicity profile was, in general, mild and manageable.

Table 1. Characteristics of the patients $(n=34)$

\begin{tabular}{|c|c|c|}
\hline & $\mathrm{n}$ & $\%$ \\
\hline \multicolumn{3}{|l|}{ Age } \\
\hline$\leq 65$ & 28 & 82.4 \\
\hline$>65$ & 6 & 17.6 \\
\hline \multicolumn{3}{|l|}{ ECOG PS } \\
\hline 0 & 5 & 14.7 \\
\hline 1 & 29 & 85.3 \\
\hline \multicolumn{3}{|l|}{ Sex } \\
\hline Female & 13 & 38.2 \\
\hline Male & 21 & 61.8 \\
\hline \multicolumn{3}{|l|}{ Smoker } \\
\hline Yes & 15 & 44.1 \\
\hline No & 18 & 52.9 \\
\hline Unknown & 1 & 2.9 \\
\hline \multicolumn{3}{|l|}{ Tumor pathological type } \\
\hline Adenocarcinoma & 32 & 94.1 \\
\hline Adenosquamous carcinoma & 2 & 5.9 \\
\hline \multicolumn{3}{|l|}{ EGFR mutation } \\
\hline Mutant & 12 & 35.3 \\
\hline Wild-type & 18 & 52.9 \\
\hline Unknown & 4 & 11.8 \\
\hline \multicolumn{3}{|l|}{ ALK translocation } \\
\hline Yes & 2 & 5.9 \\
\hline No & 17 & 50.0 \\
\hline Unknown & 15 & 44.1 \\
\hline \multicolumn{3}{|l|}{ Brain metastasis } \\
\hline Yes & 9 & 26.5 \\
\hline No & 25 & 73.5 \\
\hline \multicolumn{3}{|l|}{ Pleural metastasis } \\
\hline Yes & 17 & 50.0 \\
\hline No & 17 & 50.0 \\
\hline \multicolumn{3}{|c|}{ Previous lines of systemic therapy } \\
\hline $1-2$ & 23 & 67.6 \\
\hline$\geq 3$ & 11 & 32.4 \\
\hline \multicolumn{3}{|l|}{ Previous taxane } \\
\hline Yes & 16 & 47.1 \\
\hline No & 18 & 52.9 \\
\hline \multicolumn{3}{|l|}{ Previous EGFR/ALK TKI } \\
\hline Yes & 16 & 47.1 \\
\hline No & 18 & 52.9 \\
\hline \multicolumn{3}{|c|}{ Previous lines of chemotherapy } \\
\hline $1-2$ & 23 & 67.6 \\
\hline$\geq 3$ & 11 & 32.4 \\
\hline \multicolumn{3}{|l|}{ Previous radiotherapy } \\
\hline Yes & 11 & 32.4 \\
\hline No & 23 & 67.6 \\
\hline
\end{tabular}

Table 2. Best overall response $(n=34)$

\begin{tabular}{lll}
\hline & $\mathrm{n}$ & $\%$ \\
\hline Best overall response & & \\
Complete response & 0 & 0 \\
Partial response & 9 & 26.5 \\
Stable disease & 19 & 55.9 \\
Progressive disease & 6 & 17.6 \\
Overall response & 9 & 26.5 \\
Disease control & 28 & 82.4 \\
\hline
\end{tabular}

Table 3. Univariable analysis of factors associated with progression-free survival and overall survival

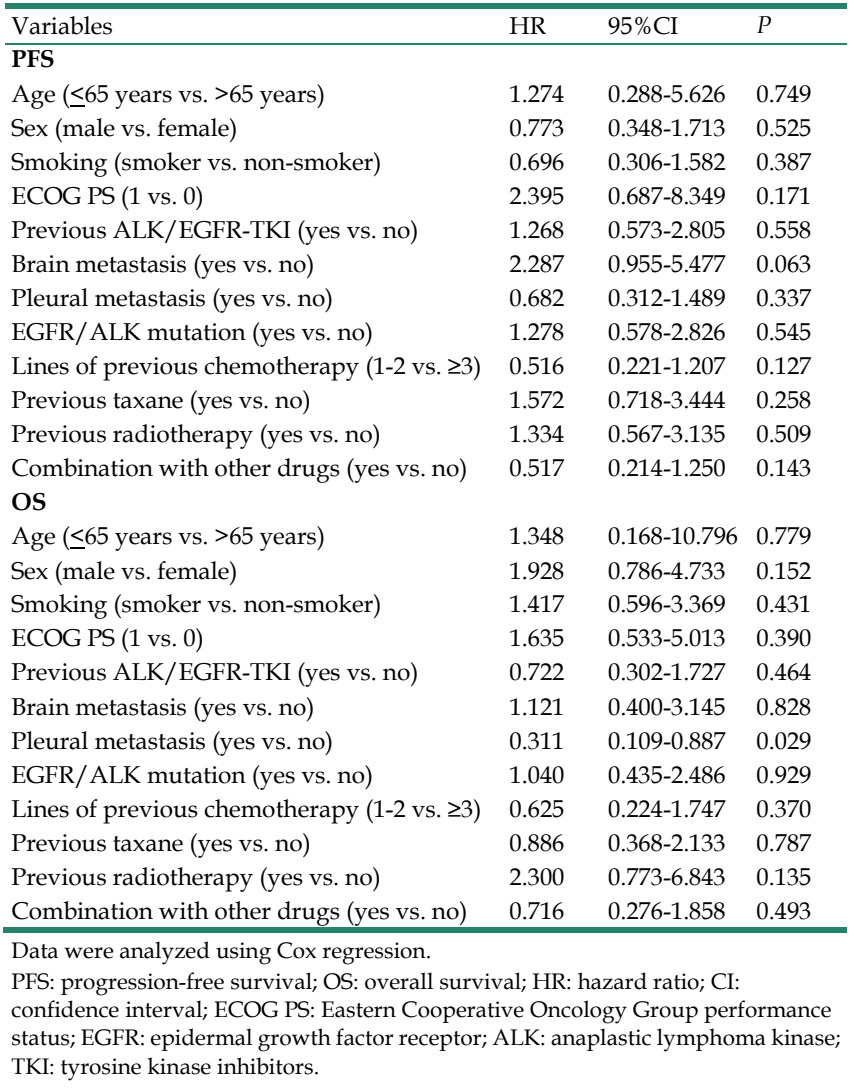

Table 4. Multivariable analysis of factors associated with progression-free survival and overall survival

\begin{tabular}{|c|c|c|c|}
\hline Variables & HR & $95 \% \mathrm{CI}$ & $P$ \\
\hline \multicolumn{4}{|l|}{ PFS } \\
\hline Brain metastasis (yes vs. no) & 1.638 & $0.525-5.111$ & 0.396 \\
\hline Lines of previous chemotherapy (1-2 vs. $\geq 3$ ) & 0.495 & $0.160-1.527$ & 0.221 \\
\hline Combination with other drugs (yes vs. no) & 0.354 & $0.134-0.935$ & 0.036 \\
\hline \multicolumn{4}{|l|}{ OS } \\
\hline ECOG PS (1 vs. 0$)$ & 2.501 & $0.660-9.475$ & 0.177 \\
\hline Sex (male vs. female) & 1.426 & $0.540-3.768$ & 0.474 \\
\hline Pleural metastasis (yes vs. no) & 0.540 & $0.118-0.980$ & 0.046 \\
\hline Previous radiotherapy (yes vs. no) & 3.768 & $0.573-6.072$ & 0.300 \\
\hline \multicolumn{4}{|c|}{$\begin{array}{l}\text { Covariates with } P \text { values }<0.20 \text { in univariable Cox proportional hazard model were } \\
\text { included in the multivariable Cox proportional hazard model. }\end{array}$} \\
\hline
\end{tabular}



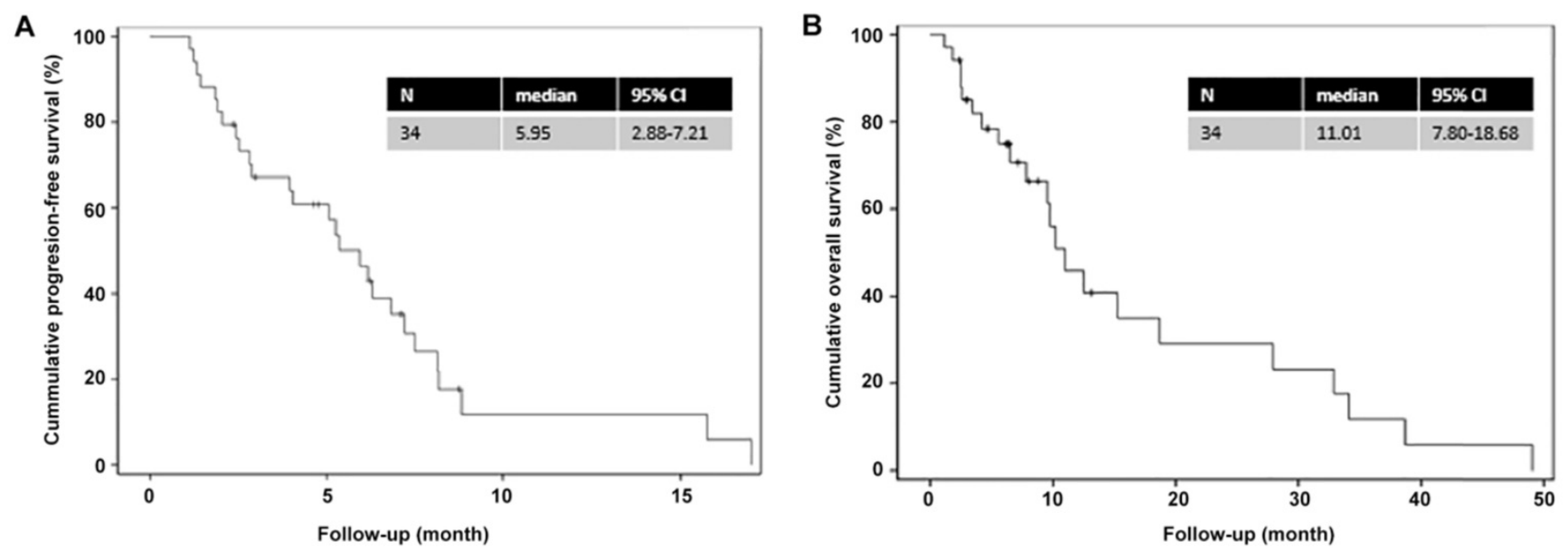

Figure 1. Kaplan-Meier curves for progression-free survival and overall survival. (A) Progression-free survival; (B) Overall survival.

Table 5. Adverse events in all patients $(n=34)$

\begin{tabular}{llllll}
\hline & Any grade & Grade 1 & Grade2 & Grade 3 Grade 4 \\
\hline Hematologic adverse events & $12(35.3)$ & $8(23.5)$ & $2(5.9)$ & $2(5.9)$ & 0 \\
Anemia & $15(44.1)$ & $7(20.6)$ & $1(2.9)$ & $6(17.6)$ & $1(2.9)$ \\
Neutropenia & $17(50.0)$ & $8(23.5)$ & $6(17.6)$ & $3(8.8)$ & 0 \\
Leukopenia & $2(5.9)$ & $2(5.9)$ & 0 & 0 & 0 \\
Thrombocytopenia & $2(5.9)$ & $2(5.9)$ & 0 & 0 & 0 \\
Hemorrhinia & 0 & 0 & 0 & 0 & 0 \\
High blood pressure & & & & & \\
Non-hematologic adverse events & $3(17.6)$ & $3(8.8)$ & $2(5.9)$ & $1(2.9)$ & 0 \\
Peripheral neurotoxicity & $17(50.0)$ & $11(32.4)$ & $6(17.6)$ & 0 & 0 \\
Vomiting & $4(11.8)$ & $3(8.8)$ & $1(2.9)$ & 0 & 0 \\
Lipsotrichia & $2(5.9)$ & $2(5.9)$ & 0 & 0 & 0 \\
Myalgia & $3(8.8)$ & $1(2.9)$ & $2(5.9)$ & 0 & 0 \\
Fatigue & $5(14.7)$ & $5(14.7)$ & 0 & 0 & 0 \\
Increased ALT/AST & 0 & 0 & 0 & 0 & 0 \\
Fever & 0 & 0 & 0 & 0 & 0 \\
Mucositis & 0 & 0 & 0 & 0 & 0 \\
Diarrhea & 0 & 0 & 0 & 0 & 0 \\
Constipation & & 0 &
\end{tabular}

All data are shown as n (\%). ALT, alanine transaminase; AST, aspartate aminotransferase.

Currently, there is no standardized regimen for the use of nab-PTX as a second or further line of treatment for NSCLC. A total dose of $260-300 \mathrm{mg} / \mathrm{m}^{2}$ of nab-PTX is usually administered for one treatment cycle of 3 weeks, with or without other anti-tumor drugs. Bevacizumab is a recognized add-on therapy for NSCLC $[15,34-36]$. To our knowledge, this is the first study that evaluated the efficacy and safety of nab-PTX in combination with bevacizumab for the treatment of patients with NSQ NSCLC after failure to at least one prior systemic regimen. The results showed that the ORR and DCR were $26.5 \%$ and $82.4 \%$, respectively; the median PFS and OS were 6.0 and 11.0 months, respectively. Previous studies of nab-PTX alone in second or further line treatment of NSCLC reported a PFS of 3.5-6.6 months and an OS of 6.8-15.7 months, with ORRs ranging from $16.1 \%-35.5 \%$ [27, 28, 37-40]. A study of nab-PTX combined with carboplatin reported a PFS of 4.0 months and an OS of 14.0 months [41]. Finally, a study of nab-PTX combined with pemetrexed showed a PFS of 4.4 months, an OS of 8.8 months, and an ORR of 14\% [42]. Three studies of PTX combined with bevacizumab in previously treated patients with NSCLC reported ORRs of $40.0 \%-48.8 \%$, DCRs of $75.0 \%-86.0 \%$, a PFS of $4.6-6.4$ months, and an OS of 9.6-14.5 months [43-45]. The treatment effects observed in the present study for the nab-PTX and bevacizumab combination were generally consistent with these previous results. The good effects of nab-PTX with bevacizumab might be due to the complementary mechanisms of the two drugs. Indeed, paclitaxel is an agent disrupting the normal functioning of the microtubules, preventing cell division and leading to apoptosis; it also has anti-angiogenic effects, probably due to the inhibition of the proliferation of endothelial cells. Bevacizumab is an anti-angiogenesis agent that directly blocks the action of VEGFR [43-45]. Animal studies showed that VEGF could reverse the effects of docetaxel, another member of the taxane family along with PTX and that the use of an anti-VEGF drug could restore the activity of docetaxel [46]. Furthermore, Shaked et al. [47] showed that PTX could induce circulating endothelial progenitor mobilization and promote angiogenesis, whereas the combined use of anti-angiogenic drugs such as bevacizumab could enhance the efficacy of PTX. Combined together, the two drugs probably have a profound synergistic effect on the tumor's blood vessels [45]. It has also been suggested that bevacizumab could normalize the tumor blood vessels, improving drug delivery and the immune microenvironment. In addition, oxygenation could be improved, preventing tumor hypoxia and hypoxia-inducible factor-mediated chemotherapy and radiotherapy resistance [48-51]. Nevertheless, beyond the clinical benefits that can be observed with the combination, the exact mechanisms for the synergy 
between bevacizumab and PTX on NSCLC and other solid tumors, in general, remain to be determined.

With regard to factors associated with patient prognosis, unexpectedly, a positive association between the presence of pleural metastasis and better OS was found. This association might be due to an improved response to the nab-PTX plus bevacizumab regimen in patients with pleural metastasis. Bevacizumab injection into the pleural cavity has been used for the management of pleural effusion and showed good benefits [52]. The combination of sb-PTX with bevacizumab has also been shown to be better than docetaxel for the management of pleural effusion from NSCLC [53]. As an anti-angiogenesis agent, bevacizumab could, at least theoretically, decrease fluid accumulation in the pleural cavity, but there are some controversies regarding this effect [54]. Alternatively, the positive association between pleural metastasis and better OS could be a result of the small sample size and sampling bias. The concomitant use of other drugs was another independent factor identified to be associated with improving survival. In the present study, all patients received nab-PTX in combination with bevacizumab, but some patients also received short courses of other concurrent anti-tumor drugs (sunitinib $(n=1)$, gemcitabine $(n=2)$, immune checkpoint inhibitor $(n=2)$, or platinum $(n=7))$, according to the treating physician's discretion and the patient's condition. Combining multiple drugs may offer the opportunity to fight the cancer cells at multiple frontlines at once, and therefore circumvent possible resistance, but at the probable cost of increased toxicity. Further trials are needed to validate the effects of multiple drug combinations.

In the present study, the main grade 3-4 AEs were neutropenia $(20.6 \%)$, leukopenia $(8.8 \%)$, and anemia (5.9\%), which can be considered as a good AE profile. This is also consistent with the known safety profiles of nab-PTX and bevacizumab [21-23, 30]. Using nab-PTX alone, the rates of grade 3-4 leukopenia, neutropenia, and anemia have been reported to be $5 \%-49 \%, 2 \%-69 \%$, and $0 \%-32 \%$ [24, 27, $28,37-40]$. In a study of nab-PTX combined with carboplatin, neutropenia, thrombocytopenia, and anemia were observed in $28.0 \%, 12.0 \%$, and $8.0 \%$ of the patients, respectively [41]. A study of nab-PTX combined with pemetrexed showed $8.2 \%$ of anemia, $6.1 \%$ of leukopenia, and $10.2 \%$ of neutropenia [42]. In two studies of sb-PTX with bevacizumab, the rates of neutropenia were $50.0 \%$ and $19.3 \%$ [53]. In PTX combined with bevacizumab, the rates of neutropenia were $20 \%-37.5 \%$ [43-45]. The toxicity profile in the present study falls within the reported ranges but in the lower end of those ranges. Nevertheless, as a retrospective study, an underreporting bias is always possible as some grade 3-4 AEs might have been treated at other hospitals.

The present study has limitations. The sample size was relatively small, and $41 \%$ of the patients had EGFR/ALK mutations. The patients received a wide variety of previous treatment regimens and concurrent supportive therapies, preventing further subgroup analyses and introducing bias. Bevacizumab was administered at two dose levels, as per physicians' choice and experience. Importantly, retrospective studies carry a risk of underreporting of the AEs. Such studies are limited to the data that can be found in the charts. Retrospective studies provide limited conclusions, but they can nevertheless provide useful insights for future clinical trials. The results should be confirmed by a multicenter study.

\section{Conclusion}

In conclusion, combined nab-PTX and bevacizumab might be an effective treatment regimen for patients with advanced NSQ NSCLC after failure of at least one prior systemic regimen, but studies have to validate those findings.

\section{Abbreviations}

NSCLC: non-small cell lung cancer; nab-PTX: nab-paclitaxel; NSQ: non-squamous; ORR: objective response rate; PFS: progression-free survival; OS: overall survival; AEs: adverse events; TKIs: Tyrosine kinase inhibitors; EGFR: epidermal growth factor receptor; ALK: anaplastic lymphoma kinase; sb-PTX: solvent-based paclitaxel; PFS: progression-free survival; VEGF: vascular epidermal growth factor; RCTs: randomized controlled trials; CAMS: Chinese Academy of Medical Sciences; PD: progressive disease; ECOG: Eastern Cooperative Oncology Group; PS: performance status; TNM: tumor node metastasis; RECIST: Response Evaluation Criteria in Solid Tumors; CR: complete response; PR: partial response; SD: stable disease; NCI-CTC: National Cancer Institute-Common Toxicity Criteria.

\section{Competing Interests}

The authors have declared that no competing interest exists.

\section{References}

1. Siegel RL, Miller KD, Jemal A. Cancer statistics, 2019. CA Cancer J Clin. 2019; 69: 7-34

2. Bray F, Ferlay J, Soerjomataram I, Siegel RL, Torre LA, Jemal A. Global cancer statistics 2018: GLOBOCAN estimates of incidence and mortality worldwide for 36 cancers in 185 countries. CA Cancer J Clin. 2018; 68: 394-424.

3. Slatore CG, Gould MK, Au DH, Deffebach ME, White E. Lung cancer stage at diagnosis: Individual associations in the prospective VITamins and lifestyle (VITAL) cohort. BMC Cancer. 2011; 11: 228. 
4. Walters S, Maringe C, Coleman MP, et al. Lung cancer survival and stage at diagnosis in Australia, Canada, Denmark, Norway, Sweden and the UK: a population-based study, 2004-2007. Thorax. 2013; 68: 551-64.

5. Chen VW, Ruiz BA, Hsieh MC, Wu XC, Ries LA, Lewis DR. Analysis of stage and clinical/prognostic factors for lung cancer from SEER registries: AJCC staging and collaborative stage data collection system. Cancer. 2014; 120 Suppl 23: 3781-92.

6. Thomas A, Rajan A, Giaccone G. Tyrosine kinase inhibitors in lung cancer. Hematol Oncol Clin North Am. 2012; 26: 589-605, viii.

7. Lindeman NI, Cagle PT, Aisner DL, et al. Updated Molecular Testing Guideline for the Selection of Lung Cancer Patients for Treatment With Targeted Tyrosine Kinase Inhibitors: Guideline From the College of American Pathologists, the International Association for the Study of Lung Cancer, and the Association for Molecular Pathology. J Thorac Oncol. 2018; 13: 323-58.

8. Kuan FC, Kuo LT, Chen MC, et al. Overall survival benefits of first-line EGFR tyrosine kinase inhibitors in EGFR-mutated non-small-cell lung cancers: a systematic review and meta-analysis. Br J Cancer. 2015; 113: 1519-28.

9. Batson S, Mitchell SA, Windisch R, Damonte E, Munk VC, Reguart N. Tyrosine kinase inhibitor combination therapy in first-line treatment of non-small-cell lung cancer: systematic review and network meta-analysis. Onco Targets Ther. 2017; 10: 2473-82.

10. Yan YF, Zheng YF, Ming PP, Deng XX, Ge W, Wu YG. Immune checkpoint inhibitors in non-small-cell lung cancer: current status and future directions. Brief Funct Genomics. 2019; 18: 147-56.

11. Pistamaltzian NF, Georgoulias V, Kotsakis A. The role of immune checkpoint inhibitors in advanced non-small cell lung cancer. Expert Rev Respir Med. 2019; 13: 435-47.

12. Thungappa S, Ferri J, Caglevic C, Passiglia F, Raez L, Rolfo C. Immune checkpoint inhibitors in lung cancer: the holy grail has not yet been found. ESMO Open. 2017; 2: e000162.

13. Gajra A, Zemla TJ, Jatoi A, et al. Time-to-Treatment-Failure and Related Outcomes Among 1000+ Advanced Non-Small Cell Lung Cancer Patients: Comparisons Between Older Versus Younger Patients (Alliance A151711). J Thorac Oncol. 2018; 13: 996-1003.

14. Besse B, Adjei A, Baas P, et al. 2nd ESMO Consensus Conference on Lung Cancer: non-small-cell lung cancer first-line/second and further lines of treatment in advanced disease. Ann Oncol. 2014; 25: 1475-84.

15. NCCN Clinical Practice Guidelines in Oncology (NCCN Guidelines). Non-Small Cell Lung Cancer. Version 6.2019. Fort Wahsington: National COmprehensive Cancer Network. 2019.

16. Goldstraw P, Chansky K, Crowley J, et al. The IASLC Lung Cancer Staging Project: Proposals for Revision of the TNM Stage Groupings in the Forthcoming (Eighth) Edition of the TNM Classification for Lung Cancer. J Thorac Oncol. 2016; 11: 39-51.

17. Cucinotto I, Fiorillo L, Gualtieri S, et al. Nanoparticle albumin bound Paclitaxel in the treatment of human cancer: nanodelivery reaches prime-time? J Drug Deliv. 2013; 2013: 905091.

18. Stinchcombe TE. Nanoparticle albumin-bound paclitaxel: a novel Cremphor-EL-free formulation of paclitaxel. Nanomedicine (Lond). 2007; 2: 415-23.

19. Chen N, Li Y, Ye Y, Palmisano M, Chopra R, Zhou S. Pharmacokinetics and pharmacodynamics of nab-paclitaxel in patients with solid tumors: disposition kinetics and pharmacology distinct from solvent-based paclitaxel. J Clin Pharmacol. 2014; 54: 1097-107.

20. Stage TB, Bergmann TK, Kroetz DL. Clinical Pharmacokinetics of Paclitaxel Monotherapy: An Updated Literature Review. Clin Pharmacokinet. 2018; 57: 7-19.

21. Sparreboom A, Baker SD, Verweij J. Paclitaxel repackaged in an albumin-stabilized nanoparticle: handy or just a dandy? J Clin Oncol. 2005; 23: 7765-7.

22. Green MR, Manikhas GM, Orlov S, et al. Abraxane, a novel Cremophor-free, albumin-bound particle form of paclitaxel for the treatment of advanced non-small-cell lung cancer. Ann Oncol. 2006; 17: 1263-8.

23. Desai N, Trieu V, Damascelli B, Soon-Shiong P. SPARC Expression Correlates with Tumor Response to Albumin-Bound Paclitaxel in Head and Neck Cancer Patients. Transl Oncol. 2009; 2: 59-64.

24. Satouchi M, Okamoto I, Sakai H, et al. Efficacy and safety of weekly nab-paclitaxel plus carboplatin in patients with advanced non-small cell lung cancer. Lung Cancer. 2013; 81: 97-101.

25. Igawa S, Nishinarita N, Takakura A, et al. Real-world evaluation of carboplatin plus a weekly dose of nab-paclitaxel for patients with advanced non-small-cell lung cancer with interstitial lung disease. Cancer Manag Res. 2018; 10: 7013-9.

26. Araya T, Kita T, Ueda T, et al. Real-World Evidence of Safety and Efficacy of Carboplatin plus Nanoparticle Albumin-Bound Paclitaxel in Patients with Advanced Non-Small-Cell Lung Cancer and Preexisting
Interstitial Lung Disease: A Retrospective Study. Can Respir J. 2019; 2019: 5315903.

27. Gong W, Sun P, Mu Z, Liu J, Yu C, Liu A. Efficacy and Safety of Nab-Paclitaxel as Second-line Chemotherapy for Locally Advanced and Metastatic Non-small Cell Lung Cancer. Anticancer Res. 2017; 37: 4687-91.

28. $\mathrm{Hu} \mathrm{W}$, Zhang Z. A phase II clinical study of using nab-paclitaxel as second-line chemotherapy for Chinese patients with advanced non-small cell lung cancer. Med Oncol. 2015; 32: 498.

29. Anzai M, Morikawa M, Okuno T, et al. Efficacy and safety of nanoparticle albumin-bound paclitaxel monotherapy as second-line therapy of cytotoxic anticancer drugs in patients with advanced non-small cell lung cancer. Medicine (Baltimore). 2017; 96: e9320.

30. Keating GM. Bevacizumab: a review of its use in advanced cancer. Drugs. 2014; 74: 1891-925.

31. Masters GA, Temin S, Azzoli CG, et al. Systemic Therapy for Stage IV Non-Small-Cell Lung Cancer: American Society of Clinical Oncology Clinical Practice Guideline Update. J Clin Oncol. 2015; 33: 3488-515.

32. Cortot AB, Audigier-Valette $C$, Molinier O, et al. Weekly paclitaxel plus bevacizumab versus docetaxel as second or third-line treatment in advanced non-squamous non-small cell lung cancer (NSCLC): Results from the phase III study IFCT-1103 ULTIMATE. J Clin Oncol. 2016; 34: 9005.

33. Oken MM, Creech RH, Tormey DC, et al. Toxicity and response criteria of the Eastern Cooperative Oncology Group. Am J Clin Oncol. 1982; 5: 649-55.

34. Russo AE, Priolo D, Antonelli G, Libra M, McCubrey JA, Ferrau F. Bevacizumab in the treatment of NSCLC: patient selection and perspectives. Lung Cancer (Auckl). 2017; 8: 259-69.

35. Gridelli C, Maione P, Rossi A, De Marinis F. The role of bevacizumab in the treatment of non-small cell lung cancer: current indications and future developments. Oncologist. 2007; 12: 1183-93.

36. Zahn MO, Linck D, Losem C, et al. AVAiLABLE NIS - AVASTIN(R) in lung cancer treatment in routine oncology practice in Germany. BMC Cancer. 2019; 19: 433

37. Jin F, Zhu H, Shi F, Kong L, Yu J. A retrospective analysis of safety and efficacy of weekly nab-paclitaxel as second-line chemotherapy in elderly patients with advanced squamous non-small-cell lung carcinoma. Clin Interv Aging. 2016; 11: 167-73.

38. Tanaka H, Taima K, Morimoto T, et al. A single-arm phase II study of nab-paclitaxel for patients with chemorefractory non-small cell lung cancer. BMC Cancer. 2017; 17: 683.

39. Duan J, Hao Y, Wan R, et al. Efficacy and safety of weekly intravenous nanoparticle albumin-bound paclitaxel for non-small cell lung cancer patients who have failed at least two prior systemic treatments. Thorac Cancer. 2017; 8: 138-46.

40. Yuan DM, Lv YL, Yao YW, et al. Efficacy and safety of Abraxane in treatment of progressive and recurrent non-small cell lung cancer patients: A retrospective clinical study. Thorac Cancer. 2012; 3: 341-7.

41. Higuchi M, Takagi H, Owada Y, et al. Efficacy and tolerability of nanoparticle albumin-bound paclitaxel in combination with carboplatin as a late-phase chemotherapy for recurrent and advanced non-small-cell lung cancer: A multi-center study of the Fukushima lung cancer association group of surgeons. Oncol Lett. 2017; 13: 4315-21.

42. Ho C, Davies AM, Sangha RS, et al. Phase I/II trial of pemetrexed plus nab-paclitaxel in advanced solid tumor patients with emphasis on non-small cell lung cancer. Invest New Drugs. 2013; 31: 1587-91.

43. Hata A, Katakami N, Tanaka K, et al. Bevacizumab plus weekly paclitaxel with or without carboplatin for previously-treated non-squamous non-small cell lung cancer. Anticancer Res. 2014; 34: 275-81.

44. Habib S, Delourme J, Dhalluin X, et al. Bevacizumab and weekly paclitaxel for non-squamous non-small cell lung cancer patients: a retrospective study. Lung Cancer. 2013; 80: 197-202.

45. Le Moulec S, Hadoux J, Gontier E, et al. Combination of paclitaxel and bevacizumab in heavily pre-treated non-small-cell lung cancer (NSCLC) patients: a case series study on 15 patients. Bull Cancer. 2013; 100: 30-7.

46. Sweeney CJ, Miller KD, Sissons SE, et al. The anti-angiogenic property of docetaxel is synergistic with a recombinant humanized monoclonal antibody against vascular endothelial growth factor or 2-methoxyestradiol but antagonized by endothelial growth factors. Cancer Res. 2001; 61: 3369-72.

47. Shaked Y, Henke E, Roodhart JM, et al. Rapid chemotherapy-induced acute endothelial progenitor cell mobilization: implications for anti-angiogenic drugs as chemosensitizing agents. Cancer Cell. 2008; 14: 263-73.

48. Goel S, Duda DG, Xu L, et al. Normalization of the vasculature for treatment of cancer and other diseases. Physiol Rev. 2011; 91: 1071-121. 
49. Jain RK. Normalizing tumor microenvironment to treat cancer: bench to bedside to biomarkers. J Clin Oncol. 2013; 31: 2205-18.

50. Carmeliet P, Jain RK. Molecular mechanisms and clinical applications of angiogenesis. Nature. 2011; 473: 298-307.

51. Jain RK. Normalization of tumor vasculature: an emerging concept in anti-angiogenic therapy. Science. 2005; 307: 58-62.

52. Zongwen S, Song K, Cong Z, Tian F, Yan Z. Evaluation of efficacy and safety for bevacizumab in treating malignant pleural effusions caused by lung cancer through intrapleural injection. Oncotarget. 2017; 8: 113318-30.

53. Qi N, Li F, Li X, Kang H, Zhao H, Du N. Combination use of paclitaxel and avastin enhances treatment effect for the NSCLC patients with malignant pleural effusion. Medicine (Baltimore). 2016; 95: e5392.

54. Sabang RL, Gandhiraj D, Fanucchi M, Epelbaum O. Role of bevacizumab in the management of the patient with malignant pleural effusion: more questions than answers. Expert Rev Respir Med. 2018; 12: 87-94. 